\title{
A Randomized Double-Blind Pilot Trial of Gabapentin Versus Placebo to Treat Alcohol Dependence and Comorbid Insomnia
}

\author{
Kirk J. Brower, Hyungjin Myra Kim, Stephen Strobbe, Maher A. Karam-Hage,
} Flavia Consens, and Robert A. Zucker

\begin{abstract}
Background: Insomnia and other sleep disturbances are common, persistent, and associated with relapse in alcohol-dependent patients. The purpose of this pilot study was to compare gabapentin versus placebo for the treatment of insomnia and prevention of relapse in alcohol-dependent patients.

Methods: Twenty-one subjects, including 10 women who met study criteria for alcohol dependence and insomnia and expressed a desire to abstain from alcohol, were recruited to the study. During a 1 to 2 week placebo lead-in and screening phase, a complete medical history, physical exam, blood tests, urine drug test, and structured interviews were performed to determine eligibility and patterns of alcohol use and sleep. Insomnia due to intoxication or acute withdrawal, psychiatric or medical illness, medications, and other sleep disorders were ruled out. Subjects were then randomized to either placebo $(n=11)$ or gabapentin $(n=10)$ for 6 weeks and titrated over a 10 -day period to $1,500 \mathrm{mg}$ or 5 pills at bedtime. After a 4-day taper, subjects were reassessed 6 weeks after ending treatment.

Results: Gabapentin significantly delayed the onset to heavy drinking, an effect which persisted for 6 weeks after treatment ended. Insomnia improved in both treatment groups during the medication phase, but gabapentin had no differential effects on sleep as measured by either subjective report or polysomnography.

Conclusion: Because gabapentin is a short-acting medication that was taken only at nighttime in this study, it may possibly exert a nocturnal effect that prevents relapse to heavy drinking by a physiological mechanism not measured in this pilot study.
\end{abstract}

Key Words: Alcohol Dependence, Insomnia, Pharmacotherapy, Gabapentin, Relapse.

I

NSOMNIA AND OTHER sleep disturbances are common, persistent, and associated with relapse in alcoholdependent patients (Brower, 2003; Feige et al., 2007). Among patients admitted for alcoholism treatment, rates of insomnia range from 36 to $91 \%$ (Brower, 2001; Cohn et al., 2003). The duration of insomnia following alcohol withdrawal may persist for 4 to 8 weeks (Alling et al.,1982) if not longer, and sleep laboratory studies utilizing polysomnography (PSG) suggest that sleep disturbances may persist for months to years during sobriety (Brower, 2001). It is hypothesized that

From the University of Michigan (KJB, HMK, SS, FC, RAZ); Addiction Research Center and Substance Abuse Section, Departments of Psychiatry (KJB, SS, RAZ) and Neurology (FC), Medical School, Ann Arbor, Michigan; Center for Statistical Consultation and Research, Department of Biostatistics, School of Public Health (HMK), Ann Arbor, Michigan; and University of Texas, Department of Behavioral Science, M.D. Anderson Cancer Center (MAK), Houston, Texas.

Received for publication July 3, 2007; accepted April 10, 2008.

Reprint requests: Kirk J. Brower, MD, University of Michigan Addiction Research Center, 4250 Plymouth Road, Ann Arbor, MI 48109-5740; Fax: 734-998-7992;E-mail:kbrower@umich.edu

Copyright (C) 2008 by the Research Society on Alcoholism.

DOI: $10.1111 /$ j.1530-0277.2008.00706.x treating sleep abnormalities in alcohol-dependent patients with medication may decrease relapse rates, but no studies have tested this hypothesis.

Several medications (e.g., disulfiram, naltrexone, acamprosate) are used to prevent relapse in alcohol-dependent patients, but very few studies have examined their effects on sleep (Snyder et al., 1981; Staner et al., 2006). Other medications such as trazodone and gabapentin are commonly prescribed for their sleep-promoting properties (Friedmann et al., 2003) and have shown some potential as sleep aids in alcohol-dependent patients in open-label studies employing subjective measures (Karam-Hage and Brower, 2000, 2003). Yet placebo-controlled studies of these medications utilizing objective sleep polysomnographic parameters are virtually unknown in alcohol-dependent patients with at least one exception (Le Bon et al., 2003). Systematically investigating the sleep effects of medications in alcohol-dependent patients could identify sleep-promoting, sleep-neutral, and sleepdisruptive agents. Knowledge of these properties could then inform clinicians about treatment options.

The purpose of this pilot study was to compare gabapentin versus placebo for the treatment of insomnia and prevention of relapse in alcohol-dependent patients. Gabapentin was chosen for a number of reasons. Open-label studies have 
demonstrated that gabapentin-treated patients with alcohol dependence and insomnia report readily measured improvement in sleep over time (Karam-Hage and Brower, 2000, 2003). Moreover, gabapentin-treated alcohol-dependent patients with insomnia were less likely to feel tired and worn out upon awakening than were trazodone-treated alcohol-dependent patients (Karam-Hage and Brower, 2003). In addition, gabapentin has been shown to improve sleep as measured by PSG, especially slow wave (deep) sleep, in healthy control subjects (Bazil et al., 2005; Foldvary-Schaefer et al., 2002; Rao et al., 1988), in patients with epilepsy (Legros and Bazil, 2003), and in patients with restless legs syndrome (Garcia-Borreguero et al., 2002). Its putative actions of enhancing GABA and modulating glutamate systems in the brain are consistent with its sleep-promoting effects (Dooley et al., 2007; Kuzniecky et al., 2002). Finally, it is well-tolerated in the presence of alcohol by healthy subjects, heavy drinkers, and alcoholics (Bazil et al., 2005; Bisaga and Evans, 2006; Myrick et al., 2007) and in patients undergoing treatment for alcohol withdrawal (Bonnet et al., 2003, 2007; Mariani et al., 2006; Voris et al., 2003). Subjects were selected for their complaints of insomnia that persisted following the period of acute alcohol withdrawal. Validated subjective and objective measures were used to measure both sleep and drinking parameters. The study hypotheses were as follows: (1) gabapentin would improve drinking outcomes compared to placebo during the 6-week medication phase, (2) gabapentin would improve symptoms of insomnia compared to placebo during the 6-week medication phase, (3) improvement in drinking outcomes would be associated with improvement in insomnia, and (4) beneficial effects of gabapentin would be sustainable for the 6-week postmedication follow-up period.

\section{MATERIALS AND METHODS}

\section{Subjects}

Twenty-one subjects, including 10 women, were recruited from either an outpatient alcohol treatment center after reviewing medical records or from the surrounding community by advertisement. The study protocol and recruitment procedures were approved by the Institutional Review Board. Consent was obtained to perform a breath alcohol test, and written informed consent for the full protocol was obtained if the blood alcohol level was $<0.05 \%$ as measured by breathalyzer.

Subjects 18 years and older were eligible if they (1) met DSM-IV criteria (American Psychiatric Association, 2000) for current alcohol dependence, (2) met study criteria for insomnia, defined as ongoing complaints of difficulty falling asleep or staying asleep, waking too early, or not feeling rested in the morning that had lasted for at least 6 months prior to the screening interview and caused significant distress or daytime impairment, and (3) expressed a desire or willingness to abstain from alcohol and other drugs (except nicotine) during the study. Women were eligible only if they were not nursing, tested negative for pregnancy, and used reliable contraception if pregnancy was possible. Insomnia was evaluated and diagnosed by one of the authors (KB, SS) via the Insomnia Interview Schedule (Morin, 1993) at a mean of 11.2 (3.0) days prior to overnight PSG. The Insomnia Interview Schedule does not require parameters such as sleep onset latency or time spent awake at night to exceed threshold values so as to diagnose insomnia.
Subjects were excluded if insomnia was due to medications or the subject required treatment with medications known to affect sleep (e.g., sedating antidepressants, anticonvulsants, antipsychotic agents, centrally acting antihistamines or antihypertensives, oral corticosteroids, sedative-hypnotics, psychomotor stimulants, or theophylline). Subjects taking medications known to influence drinking outcomes such as naltrexone, disulfiram, or acamprosate were also excluded. Subjects taking nonsedating antidepressants at a stable dose for the past 2 months with no anticipated change in dose during the course of the study were not necessarily excluded, unless the investigator determined that either the medication or the disorder for which it was prescribed was contributing to their insomnia. Three subjects qualified by taking stable doses of citalopram $20 \mathrm{mg} / \mathrm{d}$, fluoxetine $40 \mathrm{mg} / \mathrm{d}$, or sertraline $100 \mathrm{mg} / \mathrm{d}$ for at least 2 months prior to study entry. Subjects were excluded if their insomnia was due to medical illness, chronic pain, a nonalcohol substance use disorder (except nicotine dependence), or DSM-IV panic disorder, social phobia, generalized anxiety disorder, posttraumatic stress disorder, major depression, anorexia nervosa, or bulimia nervosa in the past month. A lifetime history of psychosis, bipolar disorder, or obsessive-compulsive disorder was also the cause for exclusion. Blood tests to rule out medical illness included electrolytes, glucose, thyroid stimulating hormone, renal function tests, and liver function tests.

To exclude the possibility of insomnia due to acute effects of alcohol intoxication and withdrawal, insomnia had to persist during the placebo lead-in period for at least 1 week of abstinence in the absence of withdrawal symptoms as determined by a structured withdrawal rating scale (CIWA-Ar < 8) (Sullivan et al., 1989). Other exclusionary criteria included danger to self or others, unstable or distant housing, illiteracy, cognitive impairment (Mini-Mental State Exam < 27; Folstein et al., 1975), personality disorders judged likely to interfere with compliance, known allergy or hypersensitivity to gabapentin, and impaired renal function (blood urea nitrogen or creatinine $>1.5$ times normal). Subjects were also excluded if their first night of PSG revealed evidence of a primary sleep disorder including sleep apnea (respiratory distress index $>10$ ) or periodic leg movement (PLM) disorder (PLM index with arousals $>15$ ).

Of 35 participants who underwent in-person screening and met study criteria for insomnia, 14 were excluded: 7 were lost to followup before completion of screening, 1 did not meet alcohol dependence criteria, 1 was referred for residential treatment, 1 was unable to abstain from alcohol during the screening period, 2 had positive urine drug screens for cocaine, and 2 had PSG-confirmed sleep disorders (1 with sleep apnea, 1 with PLM disorder).

\section{Procedures}

The study design consisted of (1) a screening phase of 1 to 2 weeks, including a single-blind placebo lead-in period and overnight PSG, (2) a 6-week randomized double-blind, parallel-group trial of gabapentin versus placebo, and (3) a 6-week posttrial follow-up visit. During screening, a complete medical history, physical exam, blood tests, and urine drug test were performed as well as interviews and questionnaires to determine patterns of alcohol use, sleep, craving, and mental status. During this phase, subjects were instructed to follow their usual sleep schedule and to keep a sleep diary to record the pattern of their sleep schedule prior to PSG. After completing the screening phase, qualified subjects ( $n=21$ including 10 women) were randomized to either placebo $(n=11)$ or gabapentin $(n=10)$ for 6 weeks, balanced for gender (Stout et al., 1994). The randomization schedule was generated by a statistician and implemented by a nonblinded research pharmacist. Study investigators, raters, and subjects were blinded to treatment assignment until all study visits were completed and the dataset was cleaned and locked. Subjects were assessed in-person at screening, baseline, and weeks 1 , $2,3,4,6,7,9$, and 12 . 
During the double-blind phase, study medication was titrated to 5 capsules of either gabapentin or placebo orally 45 minutes prior to bedtime over a 10-day period as tolerated. Each capsule received by the active medication group contained $300 \mathrm{mg}$ of gabapentin for a final target dose of $1,500 \mathrm{mg}$ prior to bedtime. Subjects received 1 capsule at bedtime for 2 nights, then 2 capsules for 2 nights, and finally 3 capsules for 3 nights. On day 8, subjects were reassessed by the study physician or nurse practitioner who increased the dose as tolerated to 4 capsules at bedtime for 2 nights, and then to the final dosage of 5 capsules at bedtime. Gabapentin and placebo capsules were identical in appearance and dispensed from visit to visit. After 6 weeks the medication was tapered over 4 days. Subjects also received up to six 30-minute sessions of behavioral therapy as outlined in a treatment manual with a focus to enhance adherence to study medication (Carroll and O'Malley, 1996). Behavioral therapy did not focus on sleep issues. Subjects were paid for participating in the study using a schedule that provided payments of $\$ 50$ each upon completing visits at baseline and on weeks 3, 6, and 12; as well as $\$ 25$ each for completing visits on weeks $1,2,4,7$, and 9 , for a total possible compensation of $\$ 325$.

\section{Measures}

Drinking and Other Substance Use. The Time-Line FollowBack Interview (TLFB) (Sobell et al., 1988) was used to measure the frequency and quantity of self-reported drinking during both the 90 days prior to baseline and the 12-week study period. Collateral corroboration of drinking was obtained at baseline and Week 6. Biochemical corroboration of self-reported drinking consisted of breath tests for alcohol at each study visit, and a blood level for gammaglutamyl transferase (GGT) at baseline and 6 weeks. A urine drug screen tested for amphetamines, barbiturates, cannabinoids, benzodiazepines, cocaine, and opioids. Severity of craving was measured by the Obsessive Compulsive Drinking Scale (Anton et al., 1996; Moak et al., 1998). Consequences of drinking were measured with the Short Index of Problems (Feinn et al., 2003; Miller et al., 1995). Tobacco use was compared between groups by dichotomizing subjects into smokers and nonsmokers, and averaging the number of cigarettes smoked per day as determined by the TLFB.

Subjective Sleep. The 4-item self-administered Sleep Problems Questionnaire (SPQ) (Jenkins et al., 1988) was selected for its responsiveness to improvement in sleep over 4 to 6 week intervals in alcohol-dependent patients (Karam-Hage and Brower, 2000, 2003). The 4 items ask "How often in the past month did you: (1) Have trouble falling asleep? (2) Wake up several times per night? (3) Have trouble staying asleep (including waking far too early)? (4) Wake up after your usual amount of sleep feeling tired and worn out?" Each of the 4 questions is self-rated on a scale of 0 to 5 for a maximum score of 20 , with responses coded as follows: "Not at all" $=0$, "1 to 3 days" $=1$, "4 to 7 days" $=2$, "8 to 14 days" $=3$, " 15 to 21 days" $=4$, and " 22 to 31 days" $=5$. Thus, the SPQ measures insomnia as a construct by tapping into the 4 most likely complaints of patients during a clinical encounter: early, middle and late insomnia, and sleep that is not refreshing. These complaints also correspond to the "Insomnia Type" of substance-induced sleep disorder in the DSM-IV (American Psychiatric Association, 2000). In addition, subjects completed sleep diaries on a daily basis during the medication phases (placebo lead-in and gabapentin vs. placebo), yielding weekly means for sleep onset latency (SOL), sleep efficiency (SE), wake time after sleep onset (WASO), and total sleep time (TST) (Conroy et al., 2006).

Objective Sleep. Overnight PSG was performed in the sleep laboratory on 3 occasions. Two consecutive nights occurred at the end of the placebo lead-in period. The first night served as laboratory adaptation and screening for primary sleep disorders (e.g., sleep apnea). The second night served as the baseline recording prior to the double-blind phase. The third night occurred after 3 weeks on study medication, to compare early effects of gabapentin versus placebo on objective sleep parameters. Subjects were scheduled to arrive at the University of Michigan General Clinical Research Center at 20:00 hours and had electrodes attached at 20:30 hours. Lights were typically out by 23:00 hours and on again at 07:00 hours the next morning. PSG included an electroencephalogram (C3/A2 EEG); referential electro-oculogram with electrodes placed at the outer canthus of each eye, one immediately above the other just below the horizontal plane, to record both horizontal and vertical slow and rapid eye movements (REMs); submental electromyogram (EMG); respiratory monitoring (nasal-oral thermistor monitor, abdominal and chest monitors, and ear or finger oximetry); electrocardiogram; and EMG of the anterior tibialis muscle of each leg to document PLMs. Data were recorded digitally using a paperless system (Telefactor Corp., West Conshohocken, PA) and visually scored from the digital images using standard criteria (Rechtschaffen and Kales, 1968). All records were scored by a single registered PSG technician, whose inter-rater reliability results were over $90 \%$ in our internal tests. After scoring, each PSG recording was interpreted by one of the authors (FC) who was blind to study medication group and clinical history except for age and gender. PSG parameters included TST, SOL, WASO, SE, the percentage of time spent in sleep Stages 1 (S1\%), 2 (S2\%), slow wave sleep (SWS\%) and REM sleep, and REM onset latency (REML) using standard criteria (Rechtschaffen and Kales, 1968).

Other. The Structured Clinical Interview for DSM-IV Diagnoses (First et al., 1997) was administered by trained psychiatric nurses, and used to confirm a diagnosis of current alcohol dependence (Kranzler et al., 1996b) and to rule out exclusionary DSM-IV diagnoses as listed above. Severity of anxiety and depressive symptoms was measured with the 17-item Structured Interview Guide for the Hamilton Depression Rating Scale (Williams, 1988) and the Hamilton Anxiety Rating Scale (Hamilton, 1959). Compliance with study medication was measured in all subjects using electronic pill bottle caps (MEMS TrackCap system; Aprex Corp, Fremont, CA) that recorded each time the bottle was opened (Cramer et al., 1989). At each study visit, the computerized report of opening times was reconciled with pill counts and the patient's self-report (Matsuyama et al., 1993). Blood levels of gabapentin were used as a secondary measure of compliance. Concomitant treatment, including attendance at meetings of Alcoholics Anonymous, was measured with the Project MATCH Form 90 (Miller, 1996).

\section{Analyses}

Preliminary analyses were conducted to examine the distribution of variables and to compare the gabapentin and placebo groups at baseline on demographic, substance use, sleep, and psychiatric variables. Because of small sample sizes and nonnormal distributions, continuous variables were analyzed with nonparametric statistics, and each continuous variable is represented by its median (Md) and interquartile range (IQR). An exception is data on dosage where full ranges are reported.

The primary drinking outcome variable was survival in days to first episode of heavy drinking. Heavy drinking was defined as more than 4 standard drinks in a day for women and more than 5 standard drinks in a day for men, or presenting to any study visit with a blood alcohol level $>0.08 \%$ as measured by breathalyzer. An intention-to-treat analysis was used, and any subjects lost to follow-up were categorized as relapsed to heavy drinking at the time of their last assessment. Collateral corroboration of self-reported drinking was obtained on 13 of 14 subjects who remained in the study at the 6-week follow-up. In no cases did the collateral informant report heavy drinking when the subject did not. The distribution of relapse to heavy drinking was 
compared using Kaplan-Meier survival curves and log-rank tests. Separate analyses were conducted for 6 and 12 weeks to determine if potential treatment effects persisted. A Cox regression analysis was used to control for baseline variables that correlated with time to heavy drinking in preliminary analyses. A secondary drinking outcome variable was complete abstinence from drinking alcohol during the 6-week trial.

The primary sleep variable of interest was improvement in the Sleep Problems Questionnaire (SPQ) score at 6 and 12 weeks, because of its significance in open label pilot studies (Karam-Hage and Brower, 2000, 2003). Differences in sleep outcomes were compared between treatment groups, and then used to predict heavy drinking outcomes at Week 6. Sleep diary and polysomnographic sleep variables were analyzed as secondary sleep outcomes. All tests were 2-tailed and judged significant if $p<0.05$.

\section{RESULTS}

\section{Baseline Characteristics and Correlations With Drinking Outcomes}

No significant baseline differences between groups were observed on demographic, drinking, sleep, and psychiatric measures (Tables 1 and 2). Significant correlations between baseline characteristics and time to first heavy drinking day at 12 weeks were found for age (Spearman's $r=0.44$, $p<0.05$ ) and education (Spearman's $r=0.50, p=0.02$ ). Because age and education were moderately correlated with each other (Spearman's $r=0.53, p=0.01$ ), only education was entered into the Cox regression analysis in addition to treatment group so as to limit the number of covariates given the small sample size. Results from the primary comparisons between the 2 medication groups did not change when adjusted for age, instead of education.

\section{Drinking Outcomes by Treatment Group at 6 and 12 Weeks}

Drinking outcomes during the first 6 weeks could not be verified for 6 of the 7 noncompleters ( 2 in the gabapentin group and 4 in the placebo group), so they were conservatively classified as heavy drinkers. Drinking data for the remaining noncompleter (on placebo) was obtained by a telephone interview which confirmed heavy drinking. Altogether, among the gabapentin group, $3(30.0 \%)$ of 10 were categorized as having relapsed to heavy drinking during the 6-week trial versus $9(81.8 \%)$ of 11 in the placebo group (Fisher's exact test, $p=0.03$ ). A survival analysis of time to heavy drinking by treatment group revealed a statistically significant difference ( $\log$ rank $p=0.03$ ) favoring the gabapentin group (Fig. 1). After adjusting for education, the relative risk (RR) of relapse to heavy drinking in the gabapentin group was significantly lower than that of placebo group (Cox regression, $\mathrm{RR}=0.25, p=0.047$ ). Additionally adjusting for the baseline percentage of heavy drinking days in the 6 weeks prior to screening did not change the result. Results were similar among the 14 completers.

Of the 14 patients who completed the 6-week data collection, $12(86 \%)$ patients were followed until Week 12 . The 2 patients lost to follow-up during this interval were assumed to have relapsed to heavy drinking. Using an intention-to-treat

Table 1. Baseline Characteristics by Treatment Group ${ }^{a}$

\begin{tabular}{|c|c|c|}
\hline Variables $^{\mathrm{b}}$ & Gabapentin $(n=10)$ & Placebo $(n=11)$ \\
\hline Age (year) & $46.0(30.8-60.0)$ & $44.0(41.0-54.0)$ \\
\hline Education (year) & $14.0(12.0-18.0)$ & $14.0(12.0-15.0)$ \\
\hline Male & 6 of $10(60.0 \%)$ & 5 of $11(45.5 \%)$ \\
\hline \multicolumn{3}{|l|}{ Ethnicity } \\
\hline White & 9 of $10(90.0 \%)$ & 7 of $11(63.6 \%)$ \\
\hline Black & 1 of $10(10.0 \%)$ & 1 of $11(9.1 \%)$ \\
\hline Hispanic & 0 & 2 of $11(18.2 \%)$ \\
\hline Other & 0 & 1 of $11(9.1 \%)$ \\
\hline Employed & 6 of $10(60.0 \%)$ & 8 of $11(72.7 \%)$ \\
\hline \multicolumn{3}{|l|}{ Marital status } \\
\hline Married & 6 of $10(60.0 \%)$ & 2 of $11(18.2 \%)$ \\
\hline Divorced & 2 of $10(20.0 \%)$ & 6 of $11(54.5 \%)$ \\
\hline Other & 2 of $10(20.0 \%)$ & 3 of $11(27.3 \%)$ \\
\hline Lives alone & 1 of $9(11.1 \%)$ & 3 of $11(27.3 \%)$ \\
\hline$\%$ Drinking days in past 42 days & $94.0(58.9-100.0)$ & $92.9(52.4-100.0)$ \\
\hline Drinks/drinking day in past 42 days & $4.3(3.3-12.0)$ & $7.7(4.8-10.6)$ \\
\hline$\%$ Heavy drinking days in past 42 days & $16.7(3.0-70.2)$ & $54.8(21.4-90.5)$ \\
\hline Obsessive Compulsive Drinking Scale & $9.0(5.75-20.5)$ & $12.0(6.0-14.0)$ \\
\hline Short Inventory of Problems & $19.0(7.3-35.3)$ & $30.0(20.0-38.0)$ \\
\hline Age at onset of problem drinking (yr) & $24.5(20.0-34.8)$ & $24.0(21.0-36.0)$ \\
\hline Family history positive ${ }^{c}$ & 6 of $10(60.0 \%)$ & 8 of $11(72.7 \%)$ \\
\hline Gamma-glutamyl transferase [GGT] & $43.0(37.8-90.5)$ & $50.0(22.3-170.5)$ \\
\hline Current tobacco smoker & 6 of $10(60.0 \%)$ & 3 of $11(27.3 \%)$ \\
\hline Cigarettes/day [\#] & $12.4(0.0-22.5)$ & $0.0(0.0-2.0)$ \\
\hline Hamilton Depression Rating & $6.0(3.8-8.5)$ & $8.0(6.0-11.0)$ \\
\hline Scale Hamilton Anxiety Rating Scale & $7.5(3.8-9.8)$ & $8.0(6.0-12.0)$ \\
\hline
\end{tabular}

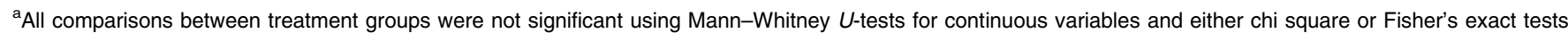
for categorical variables. Medians (interquartile ranges) are shown for continuous variables and frequencies (percentages) for discrete variables.

${ }^{b}$ Variables organized by demographic, substance-related, and psychiatric domains.

'Positive family history for alcohol problems in any first-degree relative by subjects' self-report. 


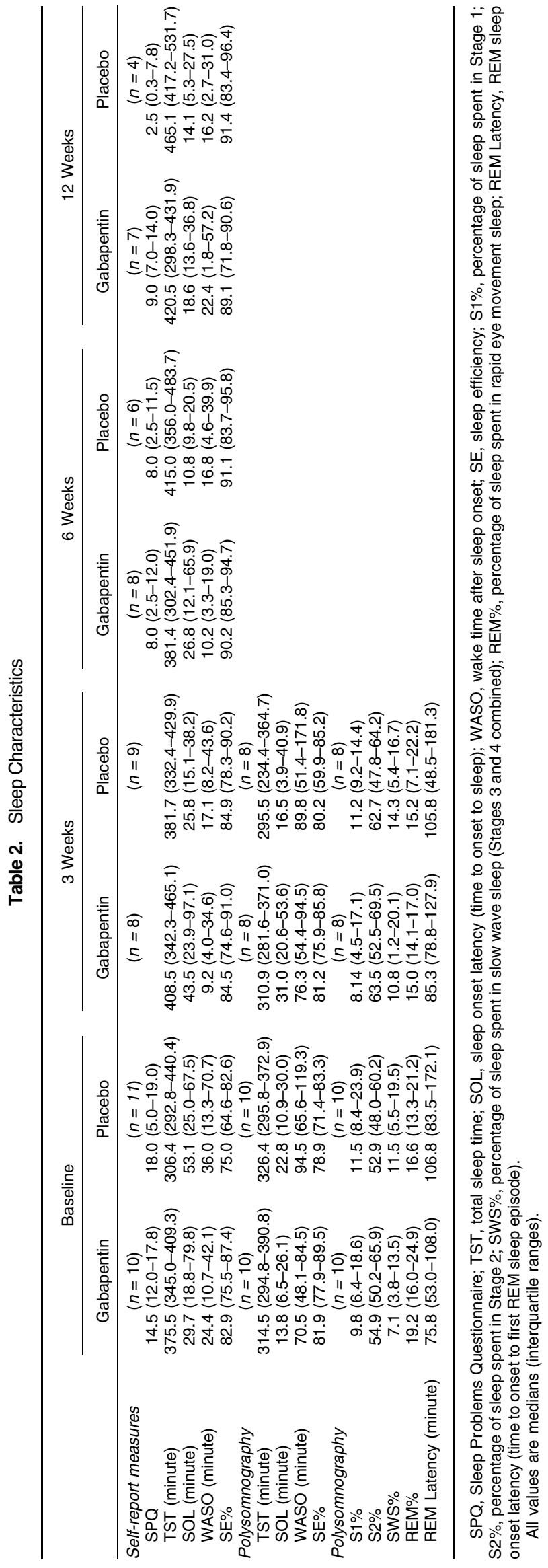

analysis, $6(60 \%)$ of 10 subjects in the gabapentin group relapsed to heavy drinking by 12 weeks versus $11(100 \%)$ of 11 in the placebo group (Fisher's exact test, $p=0.04$ ). Extending the survival analysis from baseline to 12 weeks also revealed a statistically significant difference (log rank $p=0.003$ ) favoring the gabapentin group (Fig. 1).

Although continuous abstinence through 6 weeks was more common in the gabapentin versus placebo group, the difference was not statistically significant using an intentionto-treat analysis. Altogether, $4(19.0 \%)$ of 21 subjects abstained from alcohol during the 6-week trial, including 3 $(30 \%)$ of 10 subjects in the gabapentin group and $1(9.1 \%)$ of 11 subjects in the placebo group (Fisher's exact test, $p=0.31)$. Only $1(4.8 \%)$ of 21 subjects remained continuously abstinent for 12 weeks.

\section{Sleep Outcomes by Treatment Group}

SPQ scores were available for 14 subjects at 6 weeks and 11 subjects at 12 weeks. To conduct an intention-to-treat analysis, the patients lost to follow-up by 6 weeks were assumed to have no improvement in sleep (i.e., zero change scores). Treatment group did not predict SPQ change scores from baseline to 6 weeks in either the intention-to-treat $(n=21)$ or completer $(n=14)$ analyses. In the intentionto-treat group, for example, the Md (IQR) change scores for the gabapentin and placebo groups were 5.5 (0 to 10.2) and 0 ( 0 to 7.0), respectively [ $U=46.5, Z=-0.62, p=0.53$, effect size $(E S)=0.14]$. Overall, the SPQ score improved significantly from baseline to 6 weeks in the 14 patients who completed both assessments by a Md (IQR) of 7 ( 0.8 to 11.8 ) points (Wilcoxon signed rank test for related samples: $Z=-2.91, \quad p=0.004 ; \quad \mathrm{ES}=0.55$ ). Between 6 and 12 weeks there was no overall change in SPQ scores $(n=11)$, however the difference between groups at 12 weeks showed a non-significant trend with a moderate $\mathrm{ES},(U=4.5, \mathrm{Z}=-1.80, p=0.07$, $\mathrm{ES}=0.54)$, because of worsened sleep in the gabapentin group and improved sleep in the placebo group (Table 2).

Sleep diary parameters (TST, SOL, SE, and WASO) were recorded and averaged over 1-week intervals prior to baseline, 3,6 , and 12 weeks (Table 2). To parallel the SPQ analysis during the medication phase, we tested for significant differences between treatment groups in change scores from baseline to 6 weeks $(n=14)$. Using Mann-Whitney $U$ tests, none of these differences was significant. The largest ES was observed with SOL and paradoxically favored the placebo group compared to the gabapentin group $(U=16.0$, $Z=-1.0, p=0.30, \mathrm{ES}=0.28$ ).

PSG parameters were recorded in 20 subjects at baseline (Table 2). Only one difference between groups approached significance: a more prolonged REM sleep latency was observed in the placebo group $(U=24, Z=-1.97$, $p=0.052$, ES $=0.49$ ). PSG parameters were recorded in 16 subjects at both baseline and 3 weeks (Table 2). Using a series of Wilcoxon signed rank tests, no significant main effects was 


\section{Survival Functions}

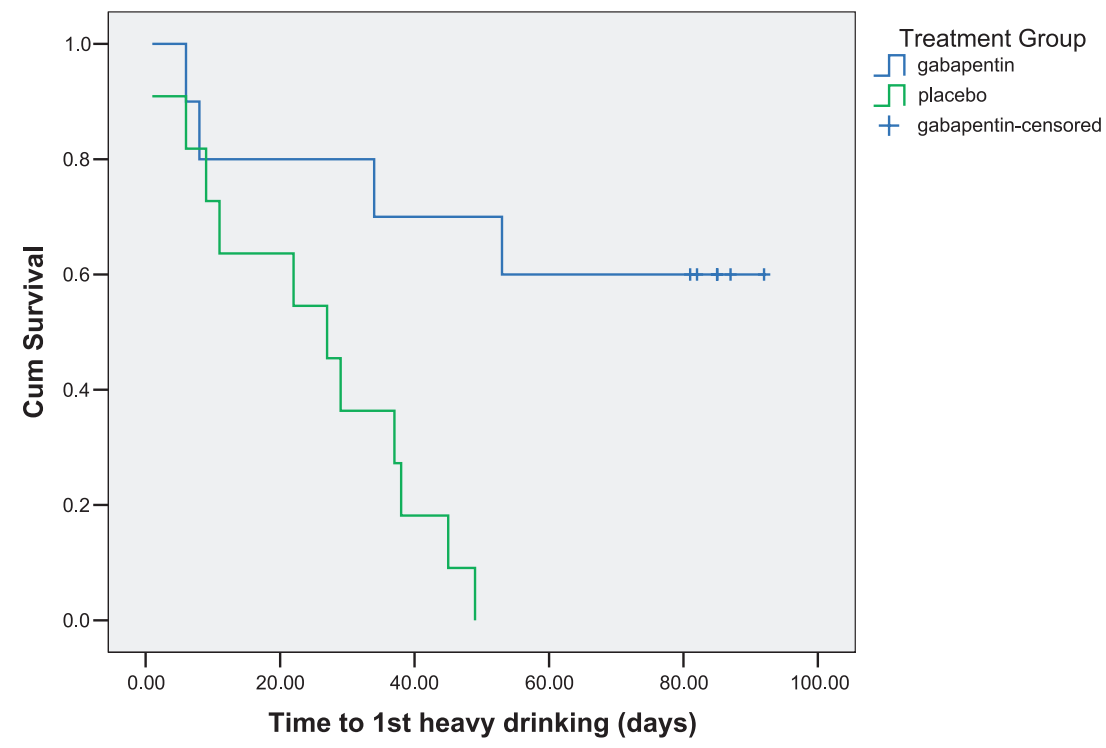

Fig. 1. Intention-to-treat $(n=21)$ Kaplan-Meier survival curves illustrating time to first day of heavy drinking from baseline to the end of both the medication trial at 42 days and the 6-week posttreatment follow-up phase at 84 days. Log-rank tests of differences between treatment groups (gabapentin vs. placebo) at both 6 and 12 weeks were statistically significant ( $p=0.03$ and $p=0.003$, respectively).

found for change over time on any of the 9 PSG parameters, with all ES $<0.30$. Moreover, treatment group did not predict changes in PSG-measured TST, SOL, SE, WASO, S1\%, $\mathrm{S} 2 \%, \mathrm{SWS} \%$, REM \%, or REML. The largest ES in these latter analyses was seen for SOL, because the gabapentin group paradoxically worsened over 3 weeks compared to the placebo group $(U=19, Z=-1.37, p=0.17, \mathrm{ES}=0.34)$.

\section{Sleep Improvement and Drinking Outcomes}

A significant relationship between sleep improvement and good drinking outcomes during the first 6 weeks was found for the 14 participants who completed the medication trial. The group that did not relapse $(n=9)$ had higher SPQ change scores than those that did relapse $(\mathrm{Md}=9.0$, $\mathrm{IQR}=5.5-15.0$ vs. $\mathrm{Md}=1.0, \mathrm{IQR}=-0.5-4.0 ; U=5.0$, $\mathrm{Z}=-2.34, p=0.019, \mathrm{ES}=0.63)$.

\section{Indicators of Study Validity}

Attrition. Seven (33\%) of 21 subjects either withdrew from the study or were lost to follow-up during the 6-week trial phase, including $2(20 \%)$ of 10 of the gabapentin group and $5(45 \%)$ of 11 of the placebo group (Fisher's exact test, $p=0.36)$. This rate of attrition was similar to other clinical trials with alcohol-dependent subjects (Kranzler et al., 1996a).

Dosing. Altogether $(n=21)$, the gabapentin group took medication over a median duration of 42 (31 to 42) days versus 39 (22 to 41) days for the placebo group $(U=30.5$, $\mathrm{Z}=-1.75, p=0.08$, $\mathrm{ES}=0.38$ ). Treatment groups did not differ significantly in terms of the prescribed dose among subjects still taking medication during Week 6 of the trial $(n=14)$. The overall median number of pills across both groups at Week 6 was 5.0 pills nightly. The range of average nightly dosing during Week 6 for the gabapentin and placebo groups was 4.1 to 5.0 pills $(1,218$ to $1,500 \mathrm{mg})$ and 3.4 to 5.0 pills, respectively.

Medication Adherence. Subjects were assessed as $100 \%$ adherent with medication if they opened their pill bottle once per night for 42 nights at approximately bedtime, and returned the correct number of fewer pills for each opening. Any deviations from this criterion (e.g., more or less openings than expected, and taking fewer or extra doses than prescribed) were discussed during compliance enhancement therapy. The following summary statistic was computed for adherence: (1) Adherence with number of doses = (\# of days that doses were verified by both MEMS cap openings and pill counts $/ 42$ days) $\times 100 \%$. Across all 21 subjects, $72.7 \%$ of doses were taken, including $69.0 \%$ of doses in the placebo group and $76.7 \%$ in the gabapentin group. Among the 14 subjects who completed the 6-week trial, 94.4\% of doses were taken. No significant differences in adherence were found between treatment groups.

Gabapentin blood levels were drawn at Week 6 and were available for 11 of 14 subjects that completed Week 6. Expected values range from 2.0 to $12.0 \mu \mathrm{g} / \mathrm{ml}$ (Mayo Medical Laboratories, Rochester, MN). The 5 subjects in the placebo group all had values of 0 except for 1 subject with $0.6 \mu \mathrm{g} / \mathrm{ml}$, which is unexplained although the limit of quantification for the assay was $0.5 \mu \mathrm{g} / \mathrm{ml}$. (After reviewing 
the source file of progress notes that were completed by the research therapist after each study visit, we concluded that actual gabapentin ingestion by this subject was highly unlikely.) The 6 subjects randomized to gabapentin all had values ranging from 2.4 to $7.1 \mu \mathrm{g} / \mathrm{ml}$, except for 1 subject with a value of 0 . Interestingly, this 1 subject relapsed to heavy drinking by 6 weeks.

Study Blind. Neither subjects nor investigators accurately predicted at the end of Week 6 which study medication the subject received, confirming the integrity of the double blind for the 13 subjects who had these data collected. Among subjects, $7(54 \%)$ of 13 were correct about which study medication they took, including $3(50 \%)$ of 6 in the placebo group and $4(57 \%)$ of 7 in the gabapentin group. The investigators were also correct in only 7 (54\%) of 13 cases.

Biochemical Corroboration of Self-Reported Drinking.

The statistic, average drinks per day, was calculated as the total number of standard drinks reported during an interval of time divided by the number of days in that interval. Blood levels of GGT correlated with average drinks per day for the 6-week period both before $(n=20$, Spearman's $r=0.54$, $p=0.014)$ and after baseline $(n=13$, Spearman's $r=0.61$, $p=0.03$ ). The average decrease in GGT blood levels from baseline to 6 weeks correlated moderately but nonsignificantly with the decrease in average drinks per day from baseline to 6 weeks for the 12 subjects who had data at both time points (Spearman's $r=0.44, p<0.15$ ). Thus, the GGT scores were consistent with levels of self-reported drinking.

Concomitant Treatment. No significant differences between treatment groups were found for concomitant interventions such as the number of Alcoholic Anonymous meetings attended.

\section{Adverse Events}

A total of 38 adverse events among 20 subjects were reported as either possibly or probably related to study medication (18 events for 10 placebo subjects and 20 events for 10 gabapentin subjects). The other 1 subject from the placebo group was lost to follow-up before the first follow-up visit. The most common side effects attributed to gabapentin versus placebo, respectively, were somnolence ( 3 subjects vs. 1 subject), headache ( 3 subjects in each group), dizziness ( 2 subjects vs. 1 subject), indigestion ( 2 vs. 4 subjects), nerve or muscle pain ( 2 subjects vs. none) and altered mental state such as feeling "spacey" or "fuzzy" (0 vs. 2 subjects). Three side effects occurred in 1 subject per group: tingling in hands, memory disturbance, and ataxia. In addition, several reported side effects each occurred in 1 gabapentin-treated subject versus no placebo subjects: dry mouth, menstrual cramps, diarrhea, constipation, muscle twitching, and paleness. Altogether 6 events, including 4 in the gabapentin group, were rated as moderate or severe; all others were rated mild. Adverse events resulted in changing the dose of medication for 1 subject receiving placebo and 3 subjects receiving gabapentin. In no cases did adverse events cause discontinuation of medication, although this information was not known for the 6 subjects that were lost to follow-up. No serious adverse events occurred.

\section{DISCUSSION}

Prior to any discussion of major findings and their potential importance, it is necessary to caution the reader that this was a pilot study with a small sample size, so the findings must be considered preliminary. That said, the major finding of this pilot study was that bedtime administration of gabapentin significantly delayed the onset of relapse (defined as the duration of time to the first heavy drinking episode) in alcoholdependent patients selected for clinical insomnia at both 6 and 12 weeks. Complete and continuous abstinence from any drinking was not associated with treatment group, but the proportion of subjects that relapsed to heavy drinking was smaller in the gabapentin versus placebo group at both 6 and 12 weeks. Thus, gabapentin had a positive effect on relapse prevention during the 6 weeks of its administration, which persisted for another 6 weeks after its treatment ended.

A second important finding was that all subjects reported improved sleep during the 6-week trial. Contrary to expectation, gabapentin did not improve SPQ sleep scores any more than placebo did during the 6 weeks of its administration. Moreover, PSG-measured sleep parameters did not show improvement from baseline to 3 weeks in either treatment group. SWS or delta sleep describes the stages of sleep (Stages 3 and 4 combined) that characterize the deepest and potentially most refreshing sleep. Although gabapentin may increase SWS\% in some populations (Bazil et al., 2005; Foldvary-Schaefer et al., 2002; Garcia-Borreguero et al., 2002; Legros and Bazil, 2003; Rao et al., 1988) during equivalent time frames, it had no significant effect on SWS\% in this short-term pilot study of alcohol-dependent individuals with insomnia.

One difference in sleep between the 2 treatment groups, although not significant $(p=0.07)$, was notable because of its moderate ES (0.54). After discontinuation of study medication, the gabapentin group had worse sleep (PSG scores) than the placebo group. The relative worsening of sleep after stopping gabapentin is suggestive of either withdrawal or a return to insomnia. Insomnia has been described in 1 case series as a gabapentin-related withdrawal symptom (Cora-Locatelli et al., 1998) and also in an 81-year-old patient after a 1-week gabapentin taper (Tran et al., 2005), but 6 weeks of persisting insomnia as a withdrawal symptom from gabapentin has not been reported previously (Norton, 2001). Thus, a return to co-occurring insomnia seems more likely as an explanation. 
The last major finding was that the participants who relapsed to heavy drinking during the 6-week trial had less improvement in insomnia than those who did not. This was an association and no inferences about causation can be made. Moreover, we did not find evidence that gabapentin's positive effect on preventing relapse was mediated by its effects on sleep, as placebo-treated subjects also had improved sleep. Nevertheless, gabapentin, which is short-acting and administered 3 to 4 times daily to treat epilepsy and neuropathic pain, was taken in this pilot study only at bedtime. Efficacy with once nightly administration is a practical advantage, because somnolence is a common side effect of gabapentin as it was in this study (Beydoun et al., 1995). Efficacy with once nightly administration may also have theoretical implications; because the mechanism by which gabapentin prevented relapse began during the nighttime without an obvious effect on sleep and then persisted during the day. This suggests some longer-acting neuromodulator effect, rather than a shorteracting effect on sleep. In other words, gabapentin may exert a nocturnal effect that improves drinking outcomes via a physiological mechanism not measured in this pilot study.

A discrepancy between subjective and objective sleep results deserves mention. At baseline both treatment groups overestimated SOL and underestimated WASO compared to PSG values (Table 2). A similar discrepancy was noted by Currie et al., 2004b) and was predictive of relapse in our sample independent of treatment group (Conroy et al., 2006). The lack of differential improvement between treatment groups in PSGrecorded WASO during the first 6 weeks may reflect the short-acting nature of gabapentin, resulting in minimal impact on nocturnal awakenings.

Gabapentin comprises a new class of medications that includes pregabalin and blocks voltage-dependent calcium channels by binding to its alpha-2-delta subunit (Stahl, 2004). It also increases cerebral GABA levels (Kuzniecky et al., 2002) and may modulate the release of glutamate and norepinephrine (Dooley et al., 2007). These effects might explain its potential to improve sleep and reduce anxiety in other studies (Foldvary-Schaefer et al., 2002; Pande et al., 1999, 2000) and to prevent relapse in this pilot study.

This is the first controlled trial to investigate the efficacy of gabapentin to improve sleep in alcohol-dependent patients. It is also the first controlled study to investigate gabapentin's efficacy to prevent relapse to heavy drinking after the acute alcohol withdrawal phase has passed. By contrast, studies of gabapentin to treat acute alcohol withdrawal reveal mixed results (Bonnet et al., 2003, 2007; Mariani et al., 2006; Voris et al., 2003) The potential efficacy of gabapentin to prevent relapse is particularly notable because it has low addictive potential [with some recently reported exceptions (Pittenger and Desan, 2007; Victorri-Vigneau et al., 2007)], does not undergo hepatic metabolism and has few interactions with other medications (Beydoun et al., 1995), is relatively safe when combined with alcohol (Bazil et al., 2005; Bisaga and Evans, 2006; Myrick et al., 2007), and is not associated with fatal overdoses when taken alone (Klein-Schwartz et al.,
2003). Gabapentin was also safe and well-tolerated in this study.

Strengths of this randomized trial included its placebo-controlled, double-blind study design, strict selection criteria to rule out comorbid conditions affecting sleep, standardized characterization of subjects across a variety of sleep and drinking variables, and intention-to-treat analyses. In addition, adherence to study medication was measured by multiple methods, biochemical corroboration of self-reported alcohol use was obtained, integrity of the study blind was maintained, and both subjective and objective sleep measures were obtained.

The major limitation of this pilot study was its small sample size which may have prevented detecting differences between treatment groups. Funding restraints prevented recruitment of a larger sample. Generalizing the results to other alcoholdependent patients, therefore, is very limited, both because of the small sample size and the strict selection criteria that were utilized. Whether gabapentin also prevents relapse to heavy drinking in alcohol-dependent patients without insomnia is unknown. All subjects in this study had insomnia. In addition, the large number of statistical tests that we conducted increased the risk of a Type I error, so that positive results may also be questioned. Nevertheless, the main positive result -increased time to relapse in the gabapentin group - was a primary outcome variable chosen a priori. The high attrition rate ( 7 of 21 subjects at 6 weeks) was another study limitation, even though it was comparable to rates observed in other clinical trials with alcohol-dependent subjects (Kranzler et al., 1996a). The schedule of subject payments for which $\$ 150$ of the total reimbursement ( $\$ 325$ ) could be obtained by week 3 of the study may have contributed to attrition at 6 and 12 weeks. In other words, subjects could obtain $\$ 150$ during the first 3 weeks, but only a total of $\$ 175$ during the next 9 weeks. Finally, subjects were allowed to sleep according to their usual schedules at home, so as to learn about the patterns of their sleep disturbance. A limitation of this methodology, however, is that some subjects manifested an irregular sleep schedule prior to their sleep laboratory studies, and this may have affected the results of their nocturnal PSG recordings. In particular, group effects could have been either amplified or diminished, particularly given the small sample size. Consequently, differential improvements in PSG parameters may have been obscured leading to false negative results. Irregular sleep patterns were not unexpected because poor sleep hygiene in alcohol-dependent patients has been previously reported (Currie et al., 2003).

Future studies should employ larger sample sizes and examine the efficacy of gabapentin for relapse prevention in the absence of insomnia, determine optimal dosing, monitor subjects for longer periods of time, and assess for discontinuation syndromes. Testing gabapentin alone and in combination with cognitive-behavioral therapy for insomnia will also be important, given the early successes of the latter in treating alcohol-dependent subjects (Arnedt et al., 2007; Currie et al., 2004a). In addition, investigating gabapentin's effects on 
mechanisms such as hyperarousal of the central nervous system (Feige et al., 2007), homeostatic sleep drive impairment (Irwin et al., 2002), and circadian rhythm abnormalities (Danel et al., 2003) may uncover nocturnal physiological effects that were not measured in this pilot study.

\section{ACKNOWLEDGMENTS}

We thank Stacy Bitterburg, Robert Fasman, Karen Kairys, and Kathleen Singer for research support, and Raymond Anton, Roseanne Armitage, Raye Litten and Timothy Roehrs for helpful comments on a draft of this article. Supported by Grant Number M01-RR000042 from the National Center for Research Resources (NCRR), a component of the National Institutes of Health (NIH), and by K24-AA00304. Its contents are solely the responsibility of the authors and do not necessarily represent the official views of NCRR or NIH. Study medication was provided by Parke-Davis (now Pfizer).

\section{REFERENCES}

Alling C, Balldin J, Bokstrom K, Gottfries CG, Karlsson I, Langstrom G (1982) Studies on duration of a late recovery period after chronic abuse of ethanol. A cross-sectional study of biochemical and psychiatric indicators. Acta Psychiatr Scand 66:384-397.

American Psychiatric Association (2000) DSM-IV-TR: Diagnostic and Statistical Manual of Mental Disorders. 4th ed. Text Revision. American Psychiatric Association, Washington DC.

Anton RF, Moak DH, Latham PK (1996) The Obsessive Compulsive Drinking Scale: a new method of assessing outcome in alcoholism treatment studies. Arch Gen Psychiatry 53:225-231.

Arnedt JT, Conroy D, Rutt J, Aloia MS, Brower KJ, Armitage R (2007) An open trial of cognitive-behavioral treatment for insomnia comorbid with alcohol dependence. Sleep Med 8:176-180.

Bazil CW, Battista J, Basner RC (2005) Gabapentin improves sleep in the presence of alcohol. J Clin Sleep Med 1:284-287.

Beydoun A, Uthman BM, Sackellares JC (1995) Gabapentin: pharmacokinetics, efficacy, and safety. Clin Neuropharmacol 18:469-481.

Bisaga A, Evans SM (2006) The acute effects of gabapentin in combination with alcohol in heavy drinkers. Drug Alcohol Depend 83:25-32.

Bonnet MH, Banger M, Leweke FM, Specka M, Muller BW, Hashemi T, Nyhuis PW, Kutscher S, Burtscheidt W, Gastpar M (2003) Treatment of acute alcohol withdrawal with gabapentin: results from a controlled two-center trial. J Clin Psychopharmacol 23:514-519.

Bonnet U, Specka M, Leweke FM, Nyhuis P, Banger M (2007) Gabapentin's acute effect on mood profile - a controlled study on patients with alcohol withdrawal. Prog Neuropsychopharmacol Biol Psychiatry 31:434 438.

Brower KJ (2001) Alcohol's effects on sleep in alcoholics. Alcohol Res Health 25:110-125.

Brower KJ (2003) Insomnia, alcoholism and relapse. Sleep Med Rev 7:523539.

Carroll K, O’Malley S (1996) Compliance Enhancement: A Manual for the Psychopharmacotherapy of Alcohol Dependence. Yale University, New Haven, CT.

Cohn TJ, Foster JH, Peters TJ (2003) Sequential studies of sleep disturbance and quality of life in abstaining alcoholics. Addiction Biology 8:455-462.

Conroy DA, Todd Arnedt J, Brower KJ, Strobbe S, Consens F, Hoffmann R, Armitage R (2006) Perception of sleep in recovering alcohol-dependent patients with insomnia: relationship with future drinking. Alcohol Clin Exp Res 30:1992-1999.

Cora-Locatelli G, Greenberg BD, Martin JD, Murphy DL (1998) Rebound psychiatric and physical symptoms after gabapentin discontinuation [letter]. J Clin Psychiatry 59:131.
Cramer JA, Mattson RH, Prevey ML, Scheyer RD, Ouellette VL (1989) How often is medication taken as prescribed? A novel assessment technique. JAMA 261:3273-3277.

Currie SR, Clark SHDC, el-Guebaly N (2004a) Randomized controlled trial of brief cognitive-behavioural interventions for insomnia in recovering alcoholics. Addiction 99:1121-1132.

Currie SR, Clark S, Rimac S, Malhotra S (2003) Comprehensive assessment of insomnia in recovering alcoholics using daily sleep diaries and ambulatory monitoring. Alcohol Clin Exp Res 27:1262-1269.

Currie SR, Malhotra S, Clark S (2004b) Agreement among subjective, objective, and collateral measures of insomnia in postwithdrawal recovering alcoholics. Behav Sleep Med 2:148-161.

Danel T, Jeanson R, Touitou Y (2003) Temporal pattern in consumption of the first drink of the day in alcohol-dependent persons. Chronobiol Int 20:1093-1102.

Dooley DJ, Taylor CP, Donevan S, Feltner D (2007) Ca2 + channel alpha2delta ligands: novel modulators of neurotransmission. Trends Pharmacol Sci 28:75-82.

Feige B, Scaal S, Hornyak M, Gann H, Riemann D (2007) Sleep electroencephalographic spectral power after withdrawal from alcohol in alcoholdependent patients. Alcohol Clin Exp Res 31:19-27.

Feinn R, Tennen H, Kranzler HR (2003) Psychometric properties of the short index of problems as a measure of recent alcohol-related problems. Alcohol Clin Exp Res 27:1436-1441.

First MB, Spitzer RL, Gibbon M, Williams JBW (1997) Structured Clinical Interview for DSM-IV Axis I Disorders (SCID-I), Clinician Version: User's Guide. American Psychiatric Press, Washington DC.

Foldvary-Schaefer N, De Leon Sanchez I, Karafa M, Mascha E, Dinner D, Morris HH (2002) Gabapentin increases slow-wave sleep in normal adults. Epilepsia 43:1493-1497.

Folstein MF, Folstein SE, McHugh PR (1975) "Mini-mental state": A practical method for grading the cognitive state of patients for the clinician. J Psychiatr Res 12:189-198.

Friedmann PD, Herman DS, Freedman S, Lemon SC, Ramsey S, Stein MD (2003) Treatment of sleep disturbance in alcohol recovery: A national survey of addiction medicine physicians. J Addict Dis 22:91-103.

Garcia-Borreguero D, Larrosa O, de la llave Y, Verger K, Masramon X, Hernandez G (2002) Treatment of restless legs syndrome with gabapentin A double-blind, cross-over study. Neurology 59:1573-1579.

Hamilton M (1959) The assessment of anxiety states by rating. Br J Med Psychol 32:50-55.

Irwin M, Gillin JC, Dang J, Weissman J, Phillips E, Ehlers CL (2002) Sleep deprivation as a probe of homeostatic sleep regulation in primary alcoholics. Biol Psychiatry 51:632-641.

Jenkins CD, Stanton BA, Niemcryk SJ, Rose RM (1988) A scale for the estimation of sleep problems in clinical research. J Clin Epidemiol 41:313-321.

Karam-Hage M, Brower KJ (2000) Gabapentin treatment for insomnia associated with alcohol dependence [letter]. Am J Psychiatry 157:51.

Karam-Hage M, Brower KJ (2003) Open pilot study of gabapentin versus trazodone to treat insomnia in alcoholic outpatients. Psychiatry Clin Neurosci 57:542-544.

Klein-Schwartz W, Shepherd JG, Gorman S, Dahl B (2003) Characterization of gabapentin overdose using a poison center case series. J Toxicol Clin Toxicol 41:11-15.

Kranzler HR, Escobar R, Lee DK, Meza E (1996a) Elevated rates of early discontinuation from pharmacotherapy trials in alcoholics and drug abusers. Alcohol Clin Exp Res 20:16-20.

Kranzler HR, Kadden RM, Babor TF, Tennen H, Rounsaville BJ (1996b) Validity of the SCID in substance abuse patients. Addiction 91:859-868.

Kuzniecky R, Ho S, Pan J, Martin R, Gilliam F, Faught E, Hetherington H (2002) Modulation of cerebral GABA by topiramate, lamotrigine, and gabapentin in healthy adults. Neurology 58:368-372.

Le Bon O, Murphy JR, Staner L, Hoffmann G, Kormoss N, Kentos M, Dupont P, Lion K, Pelc I, Verbanck P (2003) Double-blind, placebo-controlled study of the efficacy of trazodone in alcohol post-withdrawal syndrome: polysomnographic and clinical evaluations. J Clin Psychopharmacol 23:377-383. 
Legros B, Bazil CW (2003) Effects of antiepileptic drugs on sleep architecture: a pilot study. Sleep Med 4:51-55.

Mariani JJ, Rosenthal RN, Tross S, Singh P, Anand OP (2006) A randomized, open-label, controlled trial of gabapentin and phenobarbital in the treatment of alcohol withdrawal. Am J Addict 15:76-84.

Matsuyama JR, Mason BJ, Jue SG (1993) Pharmacists' interventions using an electronic medication-event monitoring device's adherence data versus pill counts. Ann Pharmacother 27:851-855.

Miller WR (1996) Form 90: A Structured Assessment Interview for Drinking and Related Behaviors. National Institute on Alcohol Abuse and Alcoholism Project MATCH Monograph Series (NIH Publication No. 96-4004), National Institutes of Health, Bethesda, MD.

Miller WR, Tonigan JS, Longabaugh R (1995) The Drinker Inventory of Consequences (DrInc): An Instrument for Assessing Adverse Consequences of Alcohol Abuse. National Institute on Alcohol Abuse and Alcoholism Project MATCH Monograph Series (NIH Publication No. 95-3911), National Institutes of Health, Bethesda, MD.

Moak DH, Anton RF, Latham PK (1998) Further validation of the Obsessive-Compulsive Drinking Scale (OCDS): relationship to alcoholism severity. Am J Addict 7:14-23.

Morin CM (1993) Insomnia: Psychological Assessment and Management. Guilford, New York.

Myrick H, Anton R, Voronin K, Wang W, Henderson S (2007) A doubleblind evaluation of gabapentin on alcohol effects and drinking in a clinical laboratory paradigm. Alcohol Clin Exp Res 31:221-227.

Norton JW (2001) Gabapentin withdrawal syndrome. Clin Neuropharmacol 24:245-246.

Pande AC, Davidson JR, Jefferson JW, Janney CA, Katzelnick DJ, Weisler RH, Greist JH, Sutherland SM (1999) Treatment of social phobia with gabapentin: a placebo-controlled study. J Clin Psychopharmacol 19:341348.

Pande AC, Pollack MH, Crockatt J, Greiner M, Chouinard G, Lydiard RB, Taylor CB, Dager SR, Shiovitz T (2000) Placebo-controlled study of gabapentin treatment of panic disorder. J Clin Psychopharmacol 20:467-471.
Pittenger C, Desan PH (2007) Gabapentin abuse, and delirium tremens upon gabapentin withdrawal. J Clin Psychiatry 68:483-484.

Rao ML, Clarenbach P, Vahlensieck M, Kratzschmar S (1988) Gabapentin augments whole blood serotonin in healthy young men. J Neural Transm 73:129-134.

Rechtschaffen A, Kales AA (1968) A Manual of Standardized Terminology, Techniques and Scoring System for Sleep Stages of Human Subjects. Government Printing Office (NIH Publication No. 204), Washington, DC.

Snyder S, Karacan I, Salis PJ (1981) Effects of disulfiram on the sleep of chronic alcoholics. Curr Alcohol 8:159-166.

Sobell LC, Sobell MB, Leo GI, Cancilla A (1988) Reliability of a timeline method: assessing normal drinkers' reports of recent drinking and a comparative evaluation across several populations. Br J Addiction 83:393-402.

Stahl SM (2004) Mechanism of action of alpha2delta ligands: voltage sensitive calcium channel (VSCC) modulators. J Clin Psychiatry 65:1033-1034.

Staner L, Boeijinga P, Danel T, Gendre I, Muzet M, Landron F, Luthringer R (2006) Effects of acamprosate on sleep during alcohol withdrawal: A double-blind placebo-controlled polysomnographic study in alcohol-dependent subjects. Alcohol Clin Exp Res 30:1492-1499.

Stout RL, Wirtz PW, Carbonari JP, Del Boca FK (1994) Ensuring balanced distribution of prognostic factors in treatment outcome research. J Stud Alcohol 12:70-75.

Sullivan JT, Sykora K, Schneiderman J, Naranjo CA, Sellers EM (1989) Assessment of alcohol withdrawal: the revised clinical institute withdrawal assessment for alcohol scale (CIWA-Ar). Br J Addict 84:1353-1357.

Tran KT, Hranicky D, Lark T, Jacob Nj (2005) Gabapentin withdrawal syndrome in the presence of a taper. Bipolar Disord 7:302-304.

Victorri-Vigneau C, Guerlais M, Jolliet P (2007) Abuse, dependency and withdrawal with gabapentin: a first case report. Pharmacopsychiatry 40:43-44.

Voris J, Smith NL, Rao SM, Thorne DL, Flowers QJ (2003) Gabapentin for the treatment of ethanol withdrawal. Substance Abuse 24:129-132.

Williams JBW (1988) A structured interview guide for the Hamilton Depression Rating Scale. Arch Gen Psychiatry 45:742-747. 